\title{
Repérer et encourager les talents prometteurs
}

\author{
Jürg Unger-Köppel \\ Dr méd., membre du Comité central, responsable du département Médecine et tarifs hospitaliers
}

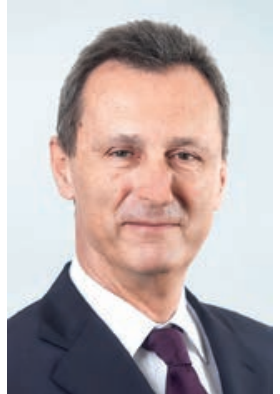

Les élections fédérales sont derrière nous. Et avec le printemps arriveront les élections à la Chambre médicale du Comité central et du président de l'ISFM. Sommes-nous prêts? Avons-nous fait le travail en amont pour pouvoir présenter des candidats adéquats? Pour trouver de bons candidats, il faut tout d'abord être au clair sur les qualités et les traits de personnalité requis. Un article de la NZZ décrivait les candidats adéquats pour un poste de codirection d'entreprise: «Il s'agit d'individus doués d'un vrai désir de coopérer, à mille lieues de toute ambition égomaniaque. De personnes capables, en dépit des divergences, de collaborer pour atteindre un objectif commun, et d'adopter un comportement égal à l'égard de tous, qu'ils jouent ou non dans la même équipe. De personnes enfin qui savent conserver une attitude de respect et de confiance, même en cas de divergence.» Ces mêmes qualités sont ce que l'on souhaiterait voir au sein des

On recherche des médecins à même d'élaborer des solutions susceptibles d'être portées par une majorité malgré des opinions opposées.

organes de direction d'associations, où l'on voit très souvent des avis diamétralement opposés s'exprimer, alors même qu'une position unifiée est vitale pour que l'organisation conserve son efficacité. Car toute divergence de vues est une faille que les adversaires vont exploiter. Or lorsque les avis s'affrontent, le silence ou les tentatives de minimiser les différences s'avèrent aussi peu efficaces qu'une attitude autoritaire. Ce qui compte alors, c'est l'aptitude à se mettre à l'écoute, à comprendre le point de vue de l'autre, et à prendre la distance nécessaire, de manière que chacun puisse céder un peu de terrain et se rallier à une solution satisfaisante pour tout le monde. Les qualités et aptitudes utiles dans le monde de l'entreprise le sont bien sûr aussi dans le cadre d'une organisation comme la nôtre. Il est important que les candidats aient prouvé, dans les activités exercées jusqu'ici, qu'ils étaient capables de collaborer de manière constructive même dans les moments difficiles. Comment reconnaît-on ce type de qualités chez un candidat? Je me ré- férerai ici à une exigence à laquelle les pédiatres se trouvent souvent confrontés, et que tous ceux qui ont travaillé dans le domaine de la protection de l'enfance comprendront. Dans ce type de contexte, on est régulièrement amené à collaborer avec de nouvelles personnes à très brève échéance. On commence avec des points de vue très éloignés, mais il faut aboutir à une décision commune, parfois lourde de conséquences, comme celle de dénoncer des parents. Seule une personne capable de garder son calme sous la pression, de réfléchir posément et d'argumenter de manière structurée peut agir efficacement dans ce domaine. D’un point de vue structurel, les séances d'une association où sont exprimées une grande diversité d'opinions ne sont pas si différentes. Ce que l'on recherche, pour siéger dans les organes de direction de nos associations, ce sont des médecins qui soient à même d'élaborer, malgré la pression d'opinions diamétralement opposées, des solutions viables, susceptibles d'être portées par une majorité. C'est ce type de représentants, aptes à coopérer, focalisés sur les solutions et clairement structurés, qu'il s'agit de repérer et d'élire. Mais suffit-il, à une époque comme la nôtre, de rester confiants et d'attendre simplement que ces candidats se présentent? Ne faudrait-il pas plutôt approcher activement les jeunes collègues qui semblent avoir ces qualités, pour les convaincre de s'engager? Nous pouvons les épauler lorsqu'ils assument leurs premières fonctions et que leur réseau s'élargit, qu'ils accumulent

\section{Une bonne préparation aux élections re-} vient donc à accompagner à plus long terme les candidats appropriés.

de l'expérience et nouent des relations en dehors de leur activité professionnelle proprement dite, les préparant à intégrer les instances centrales de notre organisation. Une bonne préparation aux élections revient donc à accompagner à plus long terme les candidats appropriés. C'est dire si le programme Coach my Career s'avère précieux non seulement pour la relève mais aussi pour une carrière au sein de notre organisation! 